\title{
Optical Carrier-Wave Subcycle Structures Associated with Supercritical Collapse of Long-Wavelength Intense Pulses Propagating in Weakly Anomalously Dispersive Media
}

\author{
A. Hofstrand and J. V. Moloney ${ }^{\circledR}$ \\ College of Optical Sciences, University of Arizona, Tucson, Arizona 85721, USA \\ and Program in Applied Mathematics, University of Arizona, Tucson, Arizona 85721, USA
}

(Received 23 August 2019; revised manuscript received 8 November 2019; published 29 January 2020)

\begin{abstract}
We predict the emergence of attosecond-duration structures on an optical carrier wave when intense, long-wavelength pulses propagate through bulk media with weak anomalous dispersion. Under certain conditions, these structures can undergo a new type of carrier-resolved supercritical collapse, forming infinite spatiotemporal gradients in the field. The mathematical conditions for the onset of this singularity are briefly overviewed, and we demonstrate with a full $3 \mathrm{D}+$ time $(3+1)$ simulation that such structures persist under realistic conditions for a 10 micron laser pulse propagating in air.
\end{abstract}

DOI: 10.1103/PhysRevLett.124.043901

In extreme nonlinear optics, broadband regions of anomalous dispersion play a critical role in achieving spatiotemporal compression of ultrashort pulses in both condensed and gaseous bulk media with wavelengths ranging from the near-infrared to long-wavelength infrared (LWIR) [1-5]. Filament propagation in such media is typically modeled by a generalized nonlinear Schrödinger equation (NLSE) [6] or its nonlinear envelope extensions [7,8]. Although when modeling femtosecond pulses at these longer wavelengths, the NLSE and its generalizations fail to accurately describe the relevant spatiotemporal reshaping of the pulse's underlying carrier field [9]. A new paradigm emerges as one transitions from a strongly dispersive, weakly nonlinear physical setting to a strongly nonlinear, weakly dispersive one as the driving laser wavelength tunes further into the transparency gap of the relevant material. Strong selfsteepening of the underlying optical carrier wave leads to shock formation [10] and, in the case of media with normal dispersion, singular gradients are regularized by harmonic walk-off that leaves a train of internally generated higher harmonics in the wake of the main pulse. This fundamental modification to modeling pulse propagation ushers in the potential for low-loss, long-range delivery of high-energy pulses over multiple Rayleigh ranges spanning multiple kilometers. The canonical mathematical description of such ultrashort pulse evolution in this long-wavelength regime is given by a generalization of the well-known KadomtsevPetviashili [11] equation (gKPE) which propagates the full field.

In this Letter, we analytically and numerically investigate the full $(3+1)$ gKPE carrier-wave-resolved model introduced in [12] for ultrashort pulses propagating through weakly dispersive media. One-dimensional, integrable [13] models of carrier-resolved pulse propagation through optical fibers with anomalous dispersion have been studied extensively [14-16]. In contrast, detailed studies of the full $(3+1)$ problem of broadband pulse propagation through bulk media with anomalous group velocity dispersion (GVD) are far from complete. Our analysis of the gKPE leads to sufficient conditions for an LWIR intense, ultrashort laser pulse propagating through the atmosphere to undergo supercritical collapse. Indeed, our model predicts a new type of optical collapse that is very different from the well-known collapsing envelope solutions of the NLSE $[17,18]$ where the underlying carrier wave is dynamically absent. We observe the formation of ultraintense, subcycle attosecond-duration spatiotemporal structures in the actual carrier field, which occur well before the self-focusing collapse point. Such extreme gradient subcycle waveforms could be used to drive quantum wave packet trajectories during tunnel ionization, to drive relativistic laser plasma interactions and laser wakefield electron acceleration, producing much shorter electron pulses than standard radio frequency accelerators. Generated electron pulses, with energy reaching the multi-GeV range, could be used to drive table-top ultrashort $\mathrm{x}$-ray sources with unprecedented brilliance and to probe photoinduced dynamics by ultrafast electron or x-ray diffraction. While our primary goal in this Letter is to identify singular ultrafast subcycle waveforms that source electron dynamics, we recognize the importance of eventually including a quantum description of the electron motion. Unfortunately, this is currently beyond the capability of existing computational resources for the situation discussed here. State of the art simulations include $(3+1)$ Maxwell coupled to a Schrödinger equation confined to extremely small micron-scale volumes limited to a $10 \mu \mathrm{m}$ propagation distance [19] and, more recently, a $(1+1)$ unidirectional propagator coupled to a $\mathrm{H}$ atom 
Schrödinger equation was simulated in [20], albeit over millimeter propagation distances. With the recent emergence of numerous experimental techniques capable of directly resolving optical carrier waves (for instance, see [21-24]), we anticipate that these subcycle structures should be readily observable, especially for the long wavelengths under consideration here. We numerically track the onset of extreme temporal and spatial (transverse) gradients as the pulse propagates, which are predicted by a rigorous virial argument. For our initial pulse to satisfy the sufficient conditions leading to this supercritical collapse, it is crucial to find media with weak and sufficiently broad regions of anomalous GVD. Finally, our predictions are validated in the realistic physical setting of atmospheric propagation by simulating the $(3+1)$-dimensional unidirectional-pulse-propagation equation (UPPE) [25].

In the case of atmospheric transmission, regions of anomalous GVD are clearly observed in the extensive HITRAN database [26] shown in Fig. 1. These regions for air are centered around approximately 4 and $10 \mu \mathrm{m}$. It is also evident from Fig. 1(b) that both of these anomalous regions are far enough removed from the absorption bands so that significant pulse power may be transmitted at these wavelengths for appropriately bandwidth-limited pulses. The anomalous region near $4 \mu \mathrm{m}$ is due to the vibrational absorption band of carbon dioxide centered at $4.26 \mu \mathrm{m}$ [seen in Fig. 1(b)]. The significantly broader anomalous region in the LWIR is located between the absorption bands of water and carbon dioxide at approximately 6.2 and $14.6 \mu \mathrm{m}$, respectively. In this Letter, we focus our attention on the anomalous, low-loss region centered around $10 \mu \mathrm{m}$, clearly observed in Figs. 1(c) and 1(d) (zoomed-in HITRAN).

Even though significant physical insight is gained by rigorously analyzing the gKPE as a model for LWIR pulse propagation through the atmosphere, one of course cannot neglect the multitude of other physical mechanisms inherent
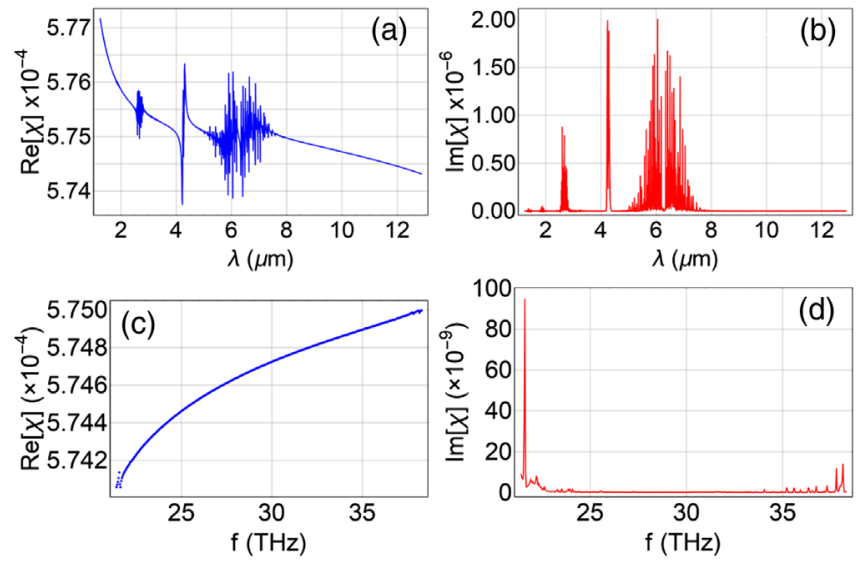

FIG. 1. Dispersion and absorption of infrared light in the atmosphere from the HITRAN database [26]. (a) Real and (b) imaginary parts of the electric susceptibility. (c), (d) are zoomed-in plots of (a) and (b) around the anomalous region centered at $10 \mu \mathrm{m}(\sim 30 \mathrm{THz})$. in the process of filamentation which are not included in our simplified gKPE model. These mechanisms include the defocusing and absorption effects of generated plasma, especially at the relatively high pulse intensities considered here, as well as the breakdown of the paraxial approximation as the beam undergoes extreme self-focusing. To study how these mechanisms influence the collapsing dynamics predicted by the gKPE model, we also simulate a $10 \mu \mathrm{m}$ pulse using the UPPE, along with the most up-to-date, inclusive model for light-matter interactions for LWIR pulses propagating through the atmosphere which has recently been experimentally validated in [27]. Remarkably, we find that the subcycle, solitonlike structures persist in the more complete UPPE simulation, although, unlike the gKPE solution, these structures do not collapse to a mathematical singularity but are eventually regularized by the pulse's interaction with generated plasma.

The gKPE (1) is our general carrier-wave-resolved model for the paraxial propagation of ultrashort laser pulses through homogeneous and isotropic, weakly dispersive materials with instantaneous Kerr nonlinearity

$\partial_{z} E+\sum_{j=1}^{N}(-1)^{j} \epsilon_{\mathrm{disp}, j} \partial_{t}^{2 j+1} E+\epsilon_{\mathrm{nl}} E^{2} \partial_{t} E=\epsilon_{\mathrm{diff}} \Delta_{\perp} \partial_{t}^{-1} E$,

where $E(z, t, \vec{x})$ is the linearly polarized electric field in normalized units propagating along the $z$ direction in a reference frame comoving with pulse's group velocity. Similar models have been proposed in [28-30]. Note, in (1), the operator $\partial_{t}^{-1}$ is defined by its Fourier transform $i / \omega$. The dimensionless parameters $\epsilon_{\mathrm{nl}}, \epsilon_{\mathrm{disp}, j}$, and $\epsilon_{\mathrm{diff}}$ correspond to the relative strengths of third-order nonlinearity, $(2 j+1)$ th-order (mixed) dispersion, and diffraction in two transverse dimensions, respectively. The explicit definitions are

$$
\begin{aligned}
\epsilon_{\mathrm{diff}} & =c /\left(2 n_{0} \omega_{0} w_{0}^{2}\right) L, \quad \epsilon_{\mathrm{disp}, j}=p_{j} \omega_{0}^{2 j+1} L, \\
\epsilon_{\mathrm{nl}} & =4 I_{0} n_{2} \omega_{0} L / c,
\end{aligned}
$$

where $c$ is the speed of light in vacuum, $\epsilon_{0}$ is the permittivity of free space, $\omega_{0}$ is the pulse's central angular frequency, $n_{0}$ is the index of refraction at the central frequency, $w_{0}$ is the initial pulse's radial width, $L$ is the propagation length scale along $z, n_{2}$ is the nonlinear index, $p_{j}$ are the polynomial-fit coefficients of $k(\omega)$ around $\omega_{0}$, and $I_{0}$ is the pulse's initial peak intensity. Various versions of the gKPE have been studied extensively in the mathematical literature [31]. A version of Eq. (1) was originally used to model the self-focusing collapse of sound in antiferromagnets [32]. If one neglects the transverse dynamics and assumes cubic dispersion, (1) becomes the well-known modified Korteweg-deVries equation (mKdVE), which can be solved exactly using the inverse 
scattering method [13]. In addition, if the medium exhibits weak anomalous dispersion, these $(1+1)$ solutions are directly related to the subcycle multisolitons first observed in the seminal work by Zabusky and Kruskal [33], with applications to shallow water waves, collisionless-plasma magnetohydrodynamic waves, and long waves in anharmonic crystals. We have found that the $(3+1)$-singular structures on the carrier wave observed in this Letter appear deeply connected to the soliton solutions of the reduced, small dispersion mKdVE. As a consequence, we can also predict that observable subcycle solitons will emerge superimposed on the optical carrier wave in $(1+1)$ waveguided structures for long-wavelength ultrashort pulses centered on spectral regions with sufficiently weak anomalous dispersion.

$$
\begin{aligned}
H[E]= & -\frac{1}{2} \sum_{j=1}^{N} \epsilon_{\mathrm{disp}, j} \int_{\mathbb{R}^{3}}\left(\partial_{t}^{j} E\right)^{2} d t d \vec{x} \\
& +\frac{1}{2} \epsilon_{\mathrm{diff}} \int_{\mathbb{R}^{3}}\left|\partial_{t}^{-1} \nabla_{\perp} E\right|^{2} d t d \vec{x} \\
& -\frac{1}{12} \epsilon_{\mathrm{nl}} \int_{\mathbb{R}^{3}} E^{4} d t d \vec{x}, \\
\mathcal{E}[E]= & \int_{\mathbb{R}^{3}} E^{2} d t d \vec{x} .
\end{aligned}
$$

Even though the classical KP equation $(N=1)$, which includes only one transverse dimension and has quadratic nonlinearity, is integrable, the integrability of equation (1) is lost in higher dimensions and for higher orders of nonlinearity or dispersion. Thus, one must rely on broader partial differential equation arguments when analyzing (1) in general. Indeed, such arguments are not easily applicable to the pseudodifferential UPPE and is the major reason for introducing the gKPE model in the first place. All of these arguments rely on the two conserved quantities given in (3), where $H$ is known as the Hamiltonian and $\mathcal{E}$ is the pulse's physical energy according to (1). These conserved quantities can be formally derived by formulating (1) via its Lagrangian and appropriately applying Noether's theorem. The constancy of both $H$ and $\mathcal{E}$ is vital to proving optical collapse. The mathematical arguments that solutions to (1) can become singular are in many ways analogous to those used to prove the envelope collapse leading to filamentation of the better-known NLSE in higher dimensions, which also has a Hamiltonian formulation. In the case of the NLSE, the Hamiltonian is expressed in terms of the field's envelope, with the inverse time derivative in (3) replaced by the fixed parameter $1 / \omega$ so that all optical carrier-related information is lost.

In the case of the gKPE, rigorous justification of sufficient conditions for the solution of (1) to become singular after a finite propagation distance is given by the so-called virial theorem $[32,34,35]$. Specifically, it states that if $H\left[E_{0}\right]$ is negative for an initial pulse in $(3+1)$ and the material's dispersive parameters are purely anomalous $\left(\epsilon_{\mathrm{disp}, j}<0\right.$ for $\left.1 \leq j \leq N\right)$, then there exists a positive, finite $Z$ such that the $L_{2}$ norm of $\nabla_{\perp} E$ goes to infinity as $z \rightarrow Z^{-}$. For the interested reader, we extend this discussion in the Supplemental Material [36] to include a more general mathematical form of (1) and explicitly state the required conditions so that the virial theorem applies. The requirement that $H\left[E_{0}\right]<0$ is contingent on the ambient medium and initial pulse properties, which we turn to now.

Here, we choose a 100 fs pulse with a temporal Ricker envelope (second-time derivative of a Gaussian) centered at $10 \mu \mathrm{m}$ over the weak anomalous window shown in Figs. 1(c) and 1(d). The Ricker waveform is spectrally close to the more-common Gaussian pulse and chosen only so that the Hamiltonian (3) can be integrated explicitly. Indeed, we have performed numerous simulations, using both Ricker and Gaussian pulses, and found very similar carrier-field evolutions. In the case of air in the LWIR, the sign of GVD is fixed over a sufficiently broad region so that only the cubic term in (1) is needed for an accurate fit over our pulse's initial bandwidth. Figure 2 shows a contour plot of the mathematical collapse point, $Z$, for this pulse (with a transverse Gaussian profile) predicted by the virial theorem as an explicit function of the initial pulse's beam waist and peak intensity. The purple-shaded region along the lower and left side of Fig. 2 represents conditions where $H\left[E_{0}\right]>0$ and so the virial theorem does not apply. As expected, Fig. 2 shows that high intensity, small beamwidth pulses collapse sooner than their less intense, larger counterparts. Actually, the point $Z$ is where the variance functional [12] vanishes and denotes an upper bound for the location of supercritical collapse. This means that as the pulse approaches the point of singularity $(\leq Z)$, the energy in the collapsing filament limits to zero linearly in $z$. Similar to the collapsing envelope of the NLSE, we find that certain solutions of the gKPE undergo supercritical collapse in $(3+1)$ and possible critical collapse in $(2+1)$ (see the Supplemental Material [36] for further details).

We now simulate this $100 \mathrm{fs}, 10 \mu \mathrm{m}$ Ricker pulse propagating through air where the rigorous and sufficient conditions for supercritical collapse have been met. In our gKPE simulation, we use the relatively high peak intensity

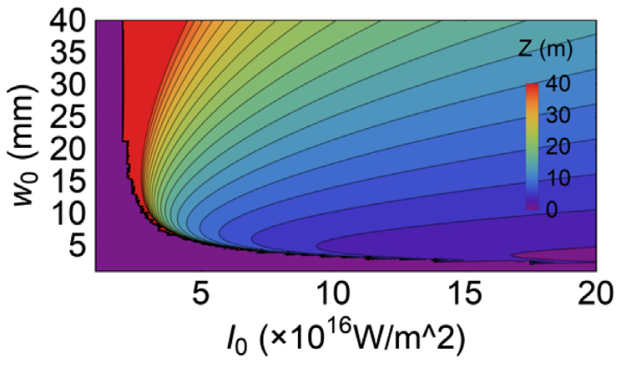

FIG. 2. Analytically obtained collapse point, $Z$, according to the virial theorem for the pulse considered here propagating through air as an explicit function of beam waist ( $e^{-2}$ radius) and initial peak intensity. 

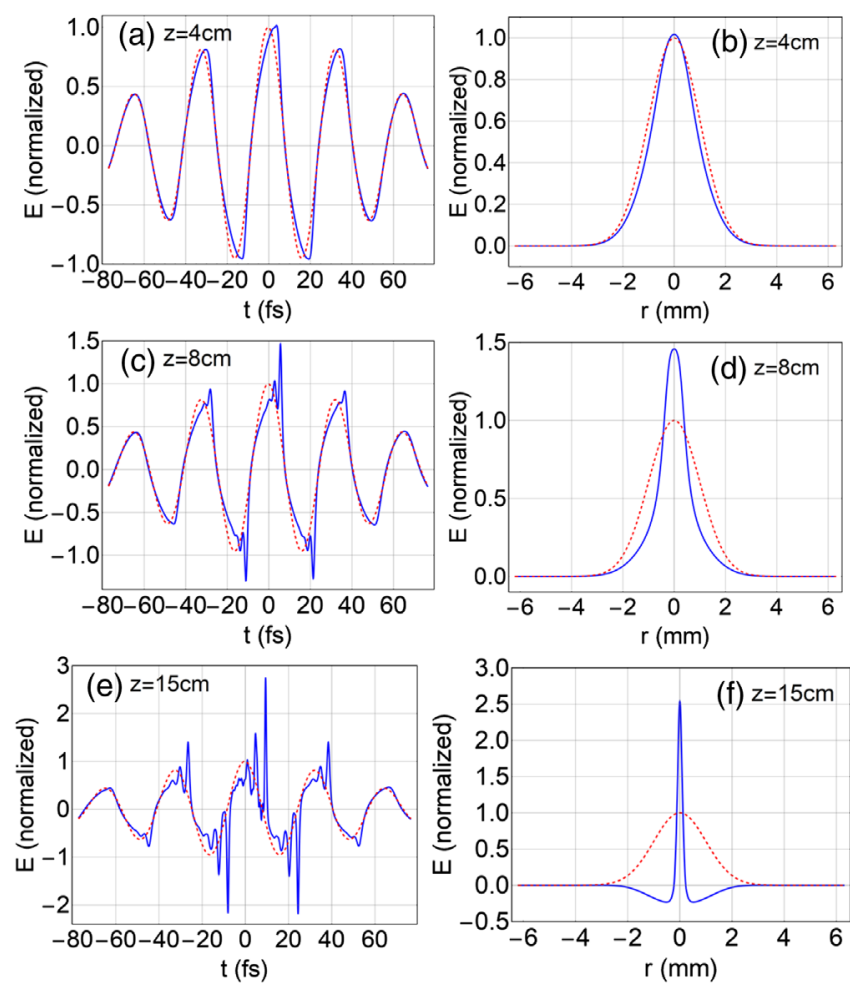

FIG. 3. Spatiotemporal reshaping (blue) of an initial $100 \mathrm{fs}$, $10 \mu \mathrm{m}$ Ricker pulse (red-dashed) propagating through air where sufficient conditions for supercritical collapse are met. The spatial profiles are taken at $t_{\max } \approx 4.0,5.8$, and $9.8 \mathrm{fs}$ in plots (b), (d), and (f), respectively.

$I_{0}=2 \times 10^{17} \mathrm{~W} \mathrm{~m}^{-2}$ and $w_{0}=2.83 \mathrm{~mm}$. As seen in Fig. 2, this pulse quickly becomes singular at or before $Z=1.6 \mathrm{~m}$. Our gKPE numerics are performed using a pseudospectral fourth-order Runge-Kutta, exponential-time-differencing) scheme introduced in [37]. Figure 3 shows the temporal, on-axis reshaping of this pulse as well as its evolving radial profile. At only $4 \mathrm{~cm}$, plots (a) and (b) in Fig. 3 show the formation of near shock fronts in the carrier wave. This initial dynamic appears to be quite generic when modeling LWIR ultrashort pulses in the weakly dispersive or strongly nonlinear regime and has been observed in [9] for the case of weak, normal GVD (see the Supplemental Material [36] for illustration of normal regularization [38,39]). As the pulse continues to propagate, the mechanism of weak anomalous GVD becomes important, and Figs. 3(c) and 3(d) show the emergence of localized structures in the actual carrier field, confined to temporal regions well below the optical cycle. After $15 \mathrm{~cm}$ of propagation, Figs. 3(e) and 3(f) show the spatiotemporal "squeezing" of these structures due to selffocusing and anomalous GVD. In this situation, the steepening gradients cannot be regularized in the gKPE model, and as expected, the field eventually becomes singular. At $15 \mathrm{~cm}$, we measure the dominant structure in Fig. 3 to have an FWHM of $0.66 \mathrm{fs}$ localized over a small fraction of a millimeter and with a peak field value greater than 2.5 times that of the initial pulse. We are unable to accurately resolve the run-away gradients in Fig. 3 past $15 \mathrm{~cm}$, given our designated error tolerance, although we expect the observed structures to indefinitely intensify and shrink in both space and time under these ideal circumstances. Additional results and details pertaining to the collapsing simulation shown in Fig. 3 are given in the Supplemental Material [36].

Finally, we display the results of a full UPPE simulation for a $100 \mathrm{fs}, 10 \mu \mathrm{m}$ temporal Gaussian pulse with $I_{0}=$ $1 \times 10^{17} \mathrm{~W} \mathrm{~m}^{-2}$ and the same initial beam waist used in Fig. 3 propagating through air. We use a lower initial peak intensity to reduce the effects of immediate photoionization. Figure 4 shows how the beam waist evolves in $z$ and displays snapshots of the on-axis field in the following three scenarios: (i) using only the purely anomalous fitted dispersion from our gKPE simulation, (ii) using the fitted anomalous dispersion with the complete effects of plasma generation included [27], and (iii) using the real (unfitted) HITRAN dispersion shown in Figs. 1(a) and 1(b) and including plasma generation. Clearly, without the regularizing effects of plasma present, Fig. 4(a) shows the pulse collapse to a point at which the numerics are unable to resolve past approximately $60 \mathrm{~cm}$. This simulation validates the singular predictions of the gKPE model in the bare UPPE computation. Figure 4(b) shows the corresponding on-axis, temporal waveform for this simulation. One can
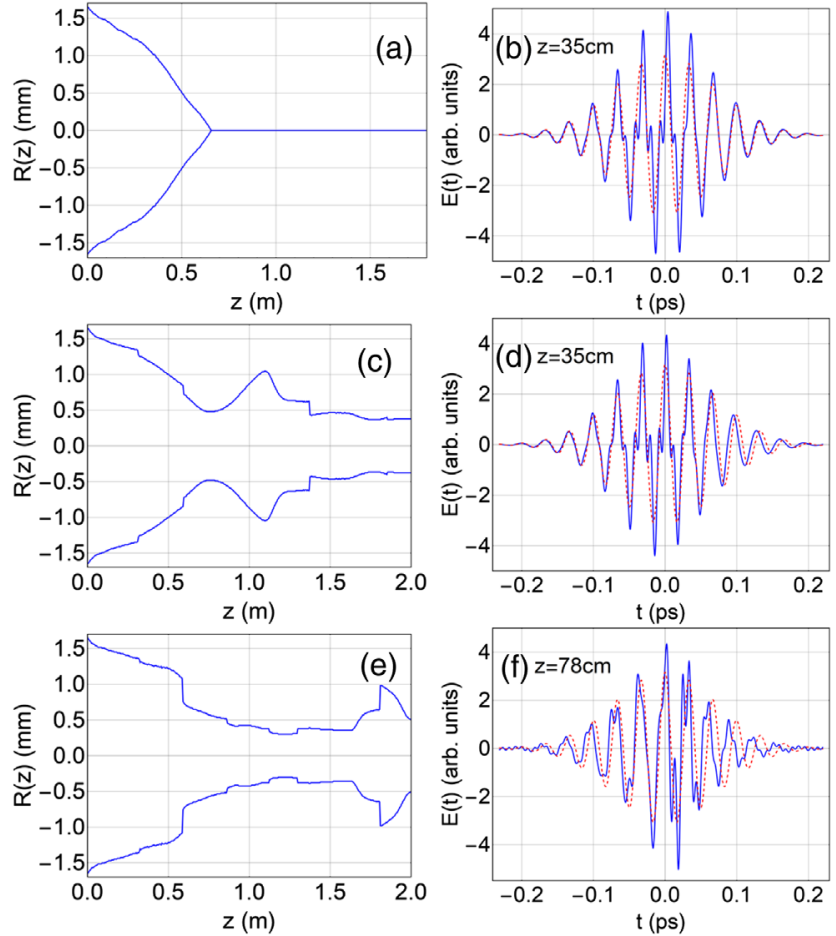

FIG. 4. The first row is a UPPE simulation using fitted (anomalous) dispersion. Plot (a) is the radial evolution, and plot (b) is the pulse's on-axis, temporal profile at a fixed $z$. The second row is a UPPE simulation of the same pulse using the same fitted dispersion, but with the effects of plasma generation included. The third row is a complete UPPE simulation of the same pulse, using the raw HITRAN data and including the effects plasma generation. 
readily observe the formation of coherent subcycle structures on the carrier wave, similar to those observed in the gKPE model. Figure 4(c) shows how the generation of plasma in the atmosphere prevents an extreme mathematical collapse from occurring although, remarkably, the subcycle structures persist in the pulse's temporal profile [Fig. 4(d)] and are present well before the nonlinear focus is reached. Furthermore, these structures are manifest even in the full HITRAN UPPE model seen in Fig. 4(f).

In conclusion, we analytically predict and numerically verify the spontaneous emergence of extreme, attosecondduration spatiotemporal singular structures within individual cycles of a long-wavelength ultrashort laser pulse propagating in a transparent nonlinear medium with a broad and weak region of anomalous dispersion. These singular structures are seen to be robust enough to persist in physical settings where plasma generation becomes significant. We anticipate that the experimental realization of these singularities will open up a whole new vista of research in extreme nonlinear optics and attosecond science. Recent developments in engineering dispersion landscapes using hollow core fibers should also greatly expand the potential for application and offer new opportunities to develop compact high power and energy sources.

This material is based upon work supported by the Air Force Office of Scientific Research under Grant No. FA9550-19-1-0032. The authors greatly appreciate technical help from P. Panagiotopoulos with Fig. 4.

[1] M. Durand, A. Jarnac, A. Houard, Y. Liu, S. Grabielle, N. Forget, A. Durécu, A. Couairon, and A. Mysyrowicz, Phys. Rev. Lett. 110, 115003 (2013).

[2] A. A. Voronin and A. M. Zheltikov, Phys. Rev. A 95, 023826 (2017).

[3] A. A. Voronin and A. M. Zheltikov, Opt. Lett. 42, 3614 (2017).

[4] D. Kartashov, S. Ališauskas, A. Pugžlys, A. Voronin, A. Zheltikov, M. Petrarca, P. Béjot, J. Kasparian, J. P. Wolf, and A. Baltuška, Opt. Lett. 38, 3194 (2013).

[5] V. Schumakova, S. Ališauskas, P. Malevich, A. A. Voronin, A. V. Mitrofanov, D. A. Sidorov-Biryukov, A. M. Zheltikov, D. Kartashov, A. Baltuška, and A. Pugžlys, Opt. Lett. 44, 2173 (2019).

[6] J. C. Diels and W. Rudolph, Ultrashort Laser Pulse Phenomena: Fundamentals, Techniques, and Applications on a Femtosecond Time Scale, 2nd ed. (Elsevier, Burlington, MA, 2006).

[7] T. Brabec and F. Krausz, Phys. Rev. Lett. 78, 3282 (1997).

[8] A. Couairon and A. Mysyrowicz, Phys. Rep. 441, 47 (2007).

[9] P. Whalen, P. Panagiotopoulos, M. Kolesik, and J. V. Moloney, Phys. Rev. A 89, 023850 (2014).

[10] P. Kinsler, J. Opt. Soc. Am. B 32, 1889 (2015).

[11] B. B. Kadomtsev and V. I. Petviashvili, Sov. Phys. Dokl. 15, 539 (1970).

[12] A. Hofstrand and J. V. Moloney, Physica (Amsterdam) 366D, 51 (2018).
[13] M. A. Ablowitz and P. A. Clarkson, Solitons, Nonlinear Evolution Equations and Inverse Scattering, London Mathematical Society Lecture Note Series, 149 (Cambridge University Press, Cambridge, United kingdom, 1992).

[14] T. Schäfer and C. Wayne, Physica (Amsterdam) 196D, 90 (2004).

[15] H. Leblond and D. Mihalache, Phys. Rev. A 79, 063835 (2009).

[16] G. Agrawal, Nonlinear Fiber Optics, 6th ed. (Elsevier, London, 2019).

[17] Y. Silberberg, Opt. Lett. 15, 1282 (1990).

[18] G. Fibich, The Nonlinear Schrödinger Equation: Singular Solutions and Optical Collapse (Springer, Switzerland, 2015).

[19] E. Lorin, S. Chelkowski, E. Zaoui, and A. Bandrauk, Physica (Amsterdam) 241D, 1059 (2012).

[20] N. Berti, P. Béjot, E. Cormier, J. Kasparian, O. Faucher, and J. P. Wolf, Phys. Rev. A 99, 061401(R) (2019).

[21] E. Goulielmakis, M. Uiberacker, R. Kienberger, A. Baltuska, V. Yakovlev, A. Scrinzi, T. Westerwalbesloh, U. Kleineberg, U. Heinzmann, M. Drescher, and F. Krausz, Science 305, 1267 (2004).

[22] C. Manzoni, O. D. Mücke, G. Cirmi, S. Fang, J. Moses, S. W. Huang, K. H. Hong, G. Cerullo, and F. X. Kärtner, Laser Photonics Rev. 9, 129 (2015).

[23] A. S. Kowligy, H. Timmers, A. J. Lind, U. Elu, F. C. Cruz, P. G. Schunemann, J. Biegert, and S. A. Diddams, Sci. Adv. 5, 2375 (2019).

[24] J. C. Travers, T. F. Grigorova, C. Brahms, and F. Belli, Nat. Photonics 13, 547 (2019).

[25] M. Kolesik, J. V. Moloney, and M. Mlejnek, Phys. Rev. Lett. 89, 283902 (2002).

[26] I. E. Gordon, L. S. Rothman et al., J. Quant. Spectrosc. Radiat. Transfer 203, 3 (2017).

[27] S. Tochitsky, E. Welch, M. Polyanskiy, I. Pogorelsky, P. Panagiotopoulos, M. Kolesik, E. M. Wright, K. S. W., J. V. Moloney, J. Pigeon, and C. Joshi, Nat. Photonics 13, 41 (2019).

[28] A. Balakin, A. Litvak, V. Mironov, and S. Skobelev, J. Exp. Theor. Phys. 104, 363 (2007).

[29] K. Glasner, M. Kolesik, J. V. Moloney, and A. C. Newell, Int. J. Opt. 2012, 868274 (2011).

[30] H. Leblond, D. Kremer, and D. Mihalache, Phys. Rev. A 81, 033824 (2010).

[31] C. Klein and J. C. Saut, arXiv:1010.5510.

[32] S. K. Turitsyn and E. G. Falkovich, Sov. Phys. JETP 62, 146 (1985).

[33] N. J. Zabusky and M. D. Kruskal, Phys. Rev. Lett. 15, 240 (1965).

[34] J. C. Saut, Indiana University mathematics Journal 42, 1011 (1993).

[35] Y. Liu, Trans. Am. Math. Soc. 353, 191 (2000).

[36] See Supplemental Material at http://link.aps.org/ supplemental/10.1103/PhysRevLett.124.043901 for more theoretical and numerical details on collapse in anomalous media and shock regularization in normal media.

[37] S. M. Cox and P. C. Matthews, J. Comput. Phys. 176, 430 (2002).

[38] H. R. Phillip and E. A. Taft, Phys. Rev. 136, A1445 (1964).

[39] J. Almeida, C. Oncebay, J. Siqueira, S. R. Muniz, L. De Boni, and C. Mendoça, Sci. Rep. 7 (2017). 